\title{
Modellistiche co-epistemologiche per la formazione del docente universitario: il progetto Prodid Uniba
}

\section{Co-epistemological modeling for academic development: the Prodid Uniba project}

\author{
Loredana Perla*, Viviana Vinci*
}

\begin{abstract}
Riassunto
Lo sviluppo professionale della docenza universitaria rappresenta una leva strategica per migliorare i risultati di apprendimento degli studenti e contribuire al progresso sociale. Le istituzioni universitarie hanno la responsabilità di sostenere - attraverso azioni di Faculty Development - processi di innovazione didattica e di qualificazione della docenza. Una delle competenze chiave da implementare nei percorsi di formazione universitari è quella relativa alla progettazione dell'insegnamento. È la stesura attenta del Syllabus che permette un lavoro di allineamento e di coerenza interna fra obiettivi formativi, risultati di apprendimento e strategie di valutazione. Si presentano i risultati di un'analisi comparativa fra Syllabi di insegnamento di area pedagogica e disciplinare (N: 94), specificatamente di quattro corsi di laurea dell'Università di Bari: due di area pedagogica (L19, LM85); due di aree disciplinari (LMG/01, L30). L'analisi comparativa dei Syllabi è stata condotta per comprendere il grado di chiarezza, coerenza e di eterogeneità nella strutturazione documentale di Ateneo e individuare elementi di criticità e aree di possibile miglioramento. L'analisi ha reso evidenti alcune risultanze capaci di orientare future prospettive di sviluppo sia a livello di indagine, che nell'ambito della formazione della docenza universitaria: fra tutte, quella di strutturare, all'interno dei Teaching and Learning Center, team interdisciplinari composti da docenti di prospettive epistemologiche differenti che possano cooperare alla redazione del Syllabus e nelle pratiche di peer learning.
\end{abstract}

\footnotetext{
${ }^{*}$ Università degli Studi di Bari Aldo Moro.

** Università Mediterranea di Reggio Calabria.

Il contributo è frutto di un lavoro condiviso. Loredana Perla è autrice dei paragrafí $1 \mathrm{e}$ 2, Viviana Vinci è autrice dei paragrafi 3, 4 e 5 . Tutti gli autori hanno contributo alla revisione del manoscritto nella sua versione finale.
}

Excellence and Innovation in Learning and Teaching (ISSNe 2499-507X), Special Issue

Doi:10.3280/exioa0-2021oa11125 
Parole Chiave: Faculty development, sviluppo professionale, Syllabus, Teaching Learning Center, team interdisciplinari

\begin{abstract}
The academic professional development represents a strategic way to improve students' learning outcomes and contribute to social progress. Universities are responsible to support teaching innovation and teaching qualification processes through Faculty Development actions. One of the key skills to be fostered in university training courses is teaching and syllabus design. It is the careful syllabus drafting that assures the alignment and internal consistency between objectives, learning outcomes and assessment strategies. The results of a comparative analysis between syllabi in the pedagogical area and subjectspecific area (N: 94) are presented, specifically from four degree courses of the University of Bari: two in the pedagogical area (L19, LM85); two in subjectspecific areas (LMG/01, L30). The comparative analysis was carried out to understand the degree of clarity, consistency and heterogeneity in the University documents and to identify critical elements and areas for possible improvement. The analysis revealed some results useful to guide future improvement strategies both at research level as well as in staff development initiatives: among all, the strategy of structuring, within the Teaching and Learning Centers, interdisciplinary teams composed of by teachers belonging to different epistemological perspectives who can cooperate in syllabus design and in peer learning practices.
\end{abstract}

Keywords: Faculty development, professional development, Syllabus, Teaching Learning Center, interdisciplinary teams

\title{
1. Qualificazione della docenza e Faculty development: volano per il miglioramento
}

L'innovazione e la qualificazione della docenza universitaria sono considerate ormai da tempo delle priorità politiche di tutti i sistemi di Higher Education a livello globale (EC, 2013; ENQA, 2015; Gaebel, \& Zhang, 2018).

Le Università, infatti, sono sempre più chiamate a rispondere a nuove sfide, quali lo sviluppo di soft skills degli studenti e l'entrata precoce nel mercato del lavoro, con conseguente riorganizzazione dei corsi di laurea nell'ottica di una maggiore coerenza con le professioni emergenti, di una maggiore razionalizzazione dei tempi e di un ripensamento degli approcci della didattica. A tal fine, 
come già indicato dal progetto Tuning (Villa Sánchez, \& Poblete Ruiz, 2008), occorre rimodulare la didattica non più in funzione dei contenuti teorico-disciplinari, ma in funzione dei risultati di apprendimento attesi, dei "learning outcomes", declinati in termini di conoscenze, capacità e competenze (Serbati, 2019).

Lo sviluppo professionale della docenza appare, in tale scenario, una leva strategica per migliorare i risultati di apprendimento degli studenti e contribuire al progresso sociale (Saryon \& Frenay, 2010; DeZure et al., 2012). La didattica universitaria rappresenta dunque l'oggetto evaluando per il miglioramento della qualità del sistema universitario (Perla, 2004): le recenti policies nazionali e internazionali evidenziano l'urgenza di costruire sistemi di garanzia dell'efficacia/efficienza del "prodotto" erogato dall'Università anche in termini di apprendimento degli studenti (insieme alla produzione della ricerca scientifica e alla terza missione).

Le istituzioni universitarie hanno la responsabilità di sostenere - attraverso azioni di Faculty Development (Sorcinelli, Austin, Eddy \& Beach, 2006; Beach, Sorcinelli, Austin \& Rivard, 2016) - un'innovazione didattica che si accompagni a processi di revisione critica e di ricerca scientifica sulle metodologie di insegnamento-apprendimento e sugli aspetti disciplinari, garantendo opportunità di sviluppo professionale e un'appropriata formazione dell'expertise didattico di alto livello (Felisatti \& Serbati, 2015, 2017). Per rispondere a tali sfide, assistiamo alla diffusione di Centri universitari per l'eccellenza nell'insegnamento e nell'apprendimento - Teaching and Learning Centers TLC - (Sorcinelli et al., 2006; Varma-Nelson \& Turner, 2017; Felisatti \& Serbati, 2017) i quali prevedono una certificazione delle competenze acquisite attraverso il percorso formativo, riconosciute e valorizzate come premialità anche ai fini carrieristici e retributivi.

Il sistema italiano di istruzione superiore, per molti anni, è apparso riluttante e incapace di sviluppare politiche efficaci per il cambiamento, all'interno di un sistema altamente centralizzato, in cui le occasioni di formazione e sviluppo professionale della docenza sono state per decenni nulle o, perlopiù, caratterizzate da approcci "frammentati": disconnessi dagli obiettivi dell'organizzazione, percepiti come un "lusso" o come un costo non necessario (anche perché non oggetto di valutazione), localizzati a livello di dipartimento e non di sistema, basati su percorsi formativi contenutistici, strettamente disciplinari. Solo assai recentemente si assiste alla diffusione di alcune esperienze di valorizzazione della qualificazione didattica universitaria, anche se lo scenario nazionale appare ancora "a macchia di leopardo", senza approcci sistemici di sviluppo professionale integrati nello sviluppo di carriera. 


\subsection{Sviluppo professionale della docenza: la sperimentazione in Uniba}

Accogliendo le sfide di rinnovamento del sistema di istruzione superiore, l'Università degli Studi di Bari ha avviato, a partire dal 2016, un protocollo di sperimentazione in quattro step:

a) adesione al progetto PRODID - Preparazione alla professionalità Docente e Innovazione Didattica, nato per iniziativa di Ettore Felisatti presso l'Università di Padova (Felisatti \& Serbati, 2015) e successivamente realizzato dalla rete di Atenei italiani (Bari, Camerino, Catania, Firenze, Foggia, Genova, Torino) costituenti l'Associazione italiana per la promozione e lo sviluppo della didattica, dell'apprendimento e dell'insegnamento in Università (ASDUNI; cfr. https://asduni.it);

b) progettazione e realizzazione del primo Teaching Learning Laboratory (TLL) istituito ufficialmente in una università pugliese (settembre 2017) con la costituzione di un Gruppo di lavoro dedicato a Faculty Development Programs;

c) strutturazione di percorsi formativi pilota - personalizzati in funzione di gruppi target differenti - per lo sviluppo professionale dei docenti universitari neo-assunti e in servizio, al fine di migliorare l'insegnamento, elevare la qualità degli apprendimenti, sostenere l'innovazione (Perla \& Vinci, 2018a, 2018b, 2020);

d) avvio di forme di co-analisi e co-progettazione con docenti afferenti ad aree disciplinari differenti, al fine di costruire - attraverso un approccio di tipo collaborativo (Perla, 2011) - un dialogo dialettico fra didattica e saperi disciplinari a livello universitario: si tratta di un lavoro co-epistemologico di ricerca (Perla, Brusa \& Vinci, 2018), di riflessione sulla propria pratica (e sull'efficacia dei mediatori utilizzati) e di formalizzazione assai utile, che consente ai docenti universitari di ripensare in profondità e riflessivamente il proprio modo di trasporre i contenuti del sapere scientifico in saperi appresi (Damiano, 2013).

\section{Analisi dei Syllabi di insegnamento: metodologia}

Uno degli obiettivi prioritari del TLL è stato effettuare un'analisi dei Syllabi di insegnamento per comprendere il grado di chiarezza e di eterogeneità nella strutturazione documentale di Ateneo, per verificare la coerenza interna fra 
obiettivi formativi e risultati di apprendimento dell'insegnamento con gli obiettivi del corso di studio, per verificare la coerenza fra obiettivi formativi e risultati di apprendimento dei diversi insegnamenti all'interno dello stesso CdS, per comprendere se vi sia una coerenza interna nell'allineamento (Biggs, 2003; O’Brein, Millis \& Cohen, 2008; Serbati \& Zaggia, 2012) fra risultati di apprendimento attesi, metodi di insegnamento, contenuti e modalità di verifica, per dedurre elementi utili alla formalizzazione di Linee Guida per la redazione efficace del Syllabus dell'insegnamento.

Per rispondere a tali obiettivi, è stata condotta un'analisi longitudinale in due fasi:

- una prima analisi, condotta nell'a.a. 2018-2019, su 288 Syllabi di insegnamento di 12 Corsi di studi sottoposti a Visita istituzionale per l'Accreditamento Periodico presso l'Università degli Studi di Bari Aldo Moro, svoltasi in data 12-16 novembre 2018 (le cui prime risultanze sono descritte in Perla \& Vinci, 2020);

- una seconda analisi, condotta nell'a.a. 2020-21 a seguito dei percorsi formativi programmati nella prima annualità di attività del TLL, su 500 Syllabi, di cui - per economia di spazi - si presenterà più in dettaglio una focalizzazione su 94 Syllabi, esemplificativa della comparazione fra Syllabi pedagogici e disciplinaristi (si veda paragrafo 3).

A livello metodologico, è stata effettuata una analisi documentale (Bowen, 2009) del corpus testuale, effettuato attraverso diversi step: a) una "scrematura", o esame superficiale, volto ad esplicitare differenze di strutture/modelli documentali emergenti e la assenza/presenza di campi documentali compilati; b) una "lettura", o esame più approfondito con analisi grounded (Strauss \& Corbin, 1990; Charmaz, 2006) di alcuni campi documentali (ad esempio, quelli dei "metodi" e della "valutazione", sottoposti a codifica assiale ed emersione di categorie semantiche a partire dalla lettura approfondita del dato testuale); c) un processo interpretativo, esito di un processo iterativo che combina analisi del contenuto e analisi tematica.

\subsection{L'analisi documentale longitudinale}

L'analisi documentale, condotta in maniera triangolata da più ricercatori (Denzin, 2010; Hussein, 2009), ha permesso:

- di far emergere un alto grado di differenziazione dei modelli documentali (alcuni strutturati secondo gli Indicatori di Dublino, altri secondo modelli 
più o meno personalizzati con aggiunta/sottrazione di voci, altri ancora specificatamente centrati sui contenuti, con differenze significative fra aree scientifico-disciplinari e fra Corsi di studio triennali, Corsi di Laurea Magistrali e Corsi Magistrali a ciclo unico);

- di individuare le maggiori criticità emergenti, afferenti in particolare l'ambito della valutazione - in cui emergono formule incentrate su "metodi/strumenti" di valutazione (colloquio orale, scritto, ecc.) e sui contenuti da dover apprendere, piuttosto che sui criteri (raramente esplicitati e, ancor più raramente, coerentemente con gli obiettivi di insegnamento e i risultati di apprendimento attesi) e sull'ambito dei metodi didattici, in cui appare prevalente l'uso esclusivo della lezione frontale accompagnata da slide; dall'analisi del contenuto è emersa inoltre una sovrapposizione semantica fra obiettivi di insegnamento e risultati di apprendimento, e fra contenuti culturali e competenze attese dello studente, con una generale attenzione più per le azioni dell'insegnante che per quelle degli studenti: la centratura, in molti casi, appare più specificatamente orientata verso la didattica disciplinare, che verso attività learning o student-centered;

- di individuare alcune differenze pre/post-formazione, ravvisabili in un generale aumento del grado di chiarezza e di uniformità nella strutturazione documentale, con un aumento significativo dei Syllabi declinati secondo i Descrittori di Dublino;

- di evidenziare con chiarezza alcune aree significative da rinforzare, le quali saranno oggetto di un percorso formativo specifico in occasione di una Summer School sull'Higher Education in programmazione presso l'Università di Bari: a) le metodologie didattiche attive - learner-centred, problem-based, experience-based - b) l'allineamento progettazione/ valutazione; c) le tecnologie didattiche.

\section{Una focalizzazione dell'analisi: comparazione dei Syllabi peda- gogici e disciplinaristi}

Si presentano i risultati parziali dell'analisi sui 500 Syllabi - i cui risultati completi saranno descritti più dettagliatamente in una pubblicazione in press focalizzando in dettaglio una comparazione fra Syllabi di insegnamenti di area pedagogica e di area disciplinare (totale: 94 Syllabi), specificatamente di quattro corsi di laurea: 
- due di area pedagogica: L19 Scienze dell'Educazione e della Formazione $(\mathrm{N}=19)$ e LM 85 Scienze Pedagogiche $(\mathrm{N}=17)$;

- due di aree disciplinari, una di ambito umanistico e una di ambito scientifico: LMG/01 Giurisprudenza $(\mathrm{N}=49)$ e L30 Fisica $(\mathrm{N}=9)$.

Obiettivo principale dell'analisi comparativa è stato l'individuazione di elementi di criticità e aree di possibile miglioramento dei Syllabi di ciascuna area, nella prospettiva di una possibile costruzione co-epistemologica di un Syllabus co-disciplinare.

Sono stati analizzati tutti i Syllabi dei quattro corsi di laurea. Per tutti i Syllabi di insegnamento, sono stati analizzati in particolare le sezioni "Metodi didattici", "Metodi di valutazione" e "Criteri di valutazione". È stata così strutturata una tabella di analisi come da esempio (Tabella 1):

Tab. 1 - Estratto dall'analisi qualitativa

\begin{tabular}{|c|c|c|c|}
\hline $\begin{array}{l}\text { Dato testuale (compilazione del campo } \\
\text { "Metodi didattici") }\end{array}$ & Memos & $\begin{array}{l}\text { Axial } \\
\text { coding }\end{array}$ & Categorie \\
\hline $\begin{array}{l}\text { Lezioni frontali e attività seminariali di } \\
\text { approfondimento su tematiche specifiche del } \\
\text { corso. }\end{array}$ & $\begin{array}{l}\text { Strutturazione: } \\
\text { Descrittori di Dublino } \\
\text { A.A.: 2018-2019 }\end{array}$ & $\begin{array}{l}\text { Lezioni } \\
\text { frontali } \\
\text { Seminari }\end{array}$ & \multirow{3}{*}{$\begin{array}{l}\text { Lezioni e } \\
\text { seminari } \\
\text { Laboratori e } \\
\text { lavori di gruppo }\end{array}$} \\
\hline $\begin{array}{l}\text { Il corso prevede lezioni frontali, lavori di } \\
\text { gruppo e seminari di approfondimento su } \\
\text { specifiche tematiche del corso. }\end{array}$ & $\begin{array}{l}\text { Strutturazione: } \\
\text { Descrittori di Dublino } \\
\text { A.A.: 2019-2020 }\end{array}$ & $\begin{array}{l}\text { Lezioni } \\
\text { frontali } \\
\text { Lavori di } \\
\text { gruppo } \\
\text { Seminari }\end{array}$ & \\
\hline $\begin{array}{l}\text { Lezioni frontali e attività laboratoriali con lavori } \\
\text { di gruppo }\end{array}$ & $\begin{array}{l}\text { Strutturazione per } \\
\text { contenuti (no Dub. } \\
\text { Des.) } \\
\text { A.A.: } 2019-2020\end{array}$ & $\begin{array}{l}\text { Lezioni } \\
\text { frontali } \\
\text { Laboratori } \\
\text { Lavori di } \\
\text { gruppo }\end{array}$ & \\
\hline
\end{tabular}

Il corpus testuale dei Syllabi analizzati per la L30 Fisica è esiguo, in quanto si tratta di un totale di 9 documenti. L'analisi documentale ha mostrato, comunque, alcune caratteristiche:

- una generale "sproporzione" a livello di compilazione fra la voce Programma - che appare come un elenco dettagliato di contenuti - e altre voci documentali (Obiettivi, Metodi e Attività di valutazione) appena accennate o assenti; 
- una particolare criticità riguardo alcuni Syllabi analizzati i quali comprendono solo indicazioni di contenuto e riferimenti bibliografici, senza specificazione di Obiettivi, Metodi e Attività di valutazione: in questi Syllabi sussiste l'assenza di qualsiasi informazione integrativa utile per lo studente;

- l'anno accademico dei Syllabi non appare sempre aggiornato;

- circa un terzo dei Syllabi presenta la declinazione dei Risultati di apprendimento attesi secondo i Descrittori di Dublino;

- i Risultati di apprendimento attesi degli studenti, in generale, sono poco definiti: ad esempio, in uno dei pochi Syllabi in cui il campo è esplicitato, compare come formula "è richiesta la dimostrazione dei teoremi scritti in grassetto";

- in alcuni Syllabi è presente, alla voce Risultati di apprendimento, la specifica: "declinare rispetto ai Descrittori di Dublino (si raccomanda che siano coerenti con i risultati di apprendimento del CdS, riportati nei quadri $A 4 a$, A4b e A4c della SUA, compreso i risultati di apprendimento trasversali)": questo dato è di un certo interesse e utilizzabile trasversalmente anche in altri Syllabi, per esplicitare il più possibile la coerenza fra i risultati di apprendimento dell'insegnamento e quelli del Corso di Studi.

Anche il corpus testuale analizzato per la L19 Scienze dell'Educazione e della Formazione mostra un certo grado di eterogeneità: il format documentale non viene sempre rispettato, alcuni Syllabi seguono una struttura più narrativa, altri presentano campi del tutto vuoti, altri ancora presentano degli "spostamenti" di informazioni, richieste in un certo campo documentale, in altri campi. Più in generale, la maggiore criticità riguarda la voce Criteri della valutazione. Il campo documentale compare solo in 1 Syllabus su 19 e in pochissimi i criteri vengono accennati nel campo Metodi di valutazione: 1'andamento prevalente vede la mancanza di esplicitazione dei criteri valutativi. Anche nella L19, come per la L30, vi è una certa difformità nell'indicazione dell'anno accademico di riferimento.

In alcuni casi la compilazione dei Risultati di apprendimento previsti è generica, priva di contestualizzazione disciplinare, come da esempio riportato (Tabella 2): 
Tab. 2 - Estratto dall'analisi dei Syllabi L-19: esempio di compilazione troppo generica

\begin{tabular}{ll}
\hline & - Capacità di comprensione e conoscenza e dei contenuti \\
& disciplinari \\
Risultati di apprendimento & - Conoscenza e capacità di comprensione applicate \\
previsti & - Autonomia di giudizio \\
& - Abilità comunicative e argomentative \\
& - Capacità di apprendere \\
\hline Contenuti di insegnamento & Elementi di Geografia generale e di Geografia umana \\
\hline
\end{tabular}

In alcuni Syllabi si denota una sovrapposizione fra campi documentali: in alcuni casi, nel campo Contenuti di insegnamento, compaiono informazioni miste, con alcuni cenni, ad esempio, ad elementi di metodo (parziali e da esplicitare meglio); in altri casi emerge una sovrapposizione fra Risultati di apprendimento previsti e Obiettivi di insegnamento; in altri ancora, fra Risultati di apprendimento previsti e Metodi didattici.

Per quanto riguarda l'analisi dei Syllabi della LM85 Scienze Pedagogiche, si osservano alcuni elementi comuni alla classe L19:

- alcuni Syllabus non sono completi;

- vi sono pochi casi in cui la compilazione dei Risultati di apprendimento pre$v i s t i$ è generico, privo di contestualizzazione disciplinare (vengono riportati i Descrittori di Dublino "vuoti"):

- si osserva un'esplicitazione dei criteri e delle modalità di valutazione maggiore nei Syllabi di area disciplinare che in quelli di area pedagogica;

- è evidente la sovrapposizione fra campi documentali, ad esempio fra Risultati di apprendimento previsti e Obiettivi di insegnamento.

Per quanto riguarda il corso LMG01 Giurisprudenza, il corpus testuale è molto più cospicuo, in quanto si tratta di 49 Syllabi, di cui uno scritto in lingua inglese. La caratteristica più evidente è che la maggior parte di questi Syllabi non è strutturata secondo i Descrittori di Dublino: prevale infatti uno schema più "narrativo".

Trentacinque Syllabi su 49 (quindi circa il 70\%) presentano, nel campo documentale dedicato ai metodi didattici, la voce Metodologia didattica convenzionale: la maggior parte in maniera vaga, senza chiarire in che modalità e con quali strumenti. La compilazione relativa ai metodi didattici appare, dunque, poco esplicativa.

Maggiore, invece, l'esplicitazione dei criteri di valutazione.

Il campo documentale Criteri di valutazione, nel corso di Giurisprudenza, è infatti quasi sempre presente (anche se, spesso, la descrizione al suo interno non 
corrisponde all'esplicitazione effettiva dei criteri valutativi, ma richiama le conoscenze disciplinari approfondite nel Programma). Fra i criteri menzionati, uno ricorrente è legato alla correttezza linguistica, terminologica, espositiva.

Come anticipato precedentemente, è stata condotta anche una codifica assiale delle categorie emergenti a seguito di procedure di analisi grounded. Come si evince dalla tabella riepilogativa successiva (Tab. 3), emerge dall'analisi un'alta differenziazione di pratiche didattiche e di valutazione (ad eccezione del corso L-30 Fisica, in cui le categorie emergenti sono inferiori, ma anche il corpus testuale è esiguo):

Tab. 3 - Axial coding: categorie differenziate per Classe di laurea

\begin{tabular}{|c|c|c|}
\hline Classe di Laurea & $\begin{array}{l}\text { 'Metodi' - Categorie } \\
\text { emergenti }\end{array}$ & 'Valutazione' - Categorie emergenti \\
\hline L-30 Fisica & $\begin{array}{l}\text { Lezioni frontali } \\
\text { Uso di slide } \\
\text { Esercitazioni } \\
\text { Esoneri } \\
\text { Lavori di gruppo } \\
\text { Peer review } \\
\text { Laboratorio }\end{array}$ & $\begin{array}{l}\text { Prova scritta } \\
\text { Prova orale } \\
\text { Accertamento delle conoscenze } \\
\text { Verifica dell'adeguatezza del linguaggio } \\
\text { Valutazione di capacità applicative delle } \\
\text { conoscenze teoriche } \\
\text { Valutazione report di laboratorio } \\
\text { Incentivazione rapidità } \\
\text { Risoluzione di problemi } \\
\text { Valutazione della scrittura di un manuale } \\
\text { procedurale }\end{array}$ \\
\hline $\begin{array}{l}\text { L-19 Scienze } \\
\text { dell'Educazione e della } \\
\text { Formazione }\end{array}$ & $\begin{array}{l}\text { Lezioni frontali } \\
\text { Seminari di approfondimento } \\
\text { Lavori di gruppo } \\
\text { Attività laboratoriali } \\
\text { Metodo deduttivo } \\
\text { Metodo induttivo } \\
\text { Metodo scientifico } \\
\text { Modalità e-learning } \\
\text { Dibattito } \\
\text { Studio di caso } \\
\text { Esercitazioni } \\
\text { Visite di studio } \\
\text { Differenziazione per i } \\
\text { frequentanti } \\
\text { Seminari integrativi } \\
\text { Proiezione di materiali } \\
\text { multimediali } \\
\text { Visione di film e documentari } \\
\text { Approfondimento con esperti } \\
\text { Uso di slide } \\
\text { Simulazioni }\end{array}$ & $\begin{array}{l}\text { Valutazione formativa } \\
\text { Prova orale } \\
\text { Prova scritta } \\
\text { Valutazione di soft skills } \\
\text { Verifica di abilità e competenze } \\
\text { professionali } \\
\text { Verifica della chiarezza espositiva e della } \\
\text { proprietà di linguaggio } \\
\text { Accertamento delle conoscenze } \\
\text { Verifica della comprensione concettuale } \\
\text { Prove individuali e di gruppo } \\
\text { Differenziazione delle prove per non } \\
\text { frequentanti } \\
\text { Prova di comprensione di testo } \\
\text { specialistico } \\
\text { Esoneri su parti di programma } \\
\text { Progetto osservativo } \\
\text { Verifica della capacità di rielaborazione } \\
\text { personale }\end{array}$ \\
\hline
\end{tabular}




\begin{tabular}{|c|c|c|}
\hline & $\begin{array}{l}\text { Didattica per progetti } \\
\text { Flipped classroom } \\
\text { Lezione dialogata } \\
\text { Osservazioni in contesti reali } \\
\text { Partecipazione ad attività di } \\
\text { ricerca } \\
\text { Materiali bibliografici di } \\
\text { approfondimento } \\
\text { Social network }\end{array}$ & \\
\hline $\begin{array}{l}\text { LM-85 Scienze } \\
\text { Pedagogiche }\end{array}$ & $\begin{array}{l}\text { Lezioni frontali } \\
\text { Laboratori } \\
\text { Seminari } \\
\text { Supporti multimediali } \\
\text { Presentazioni degli studenti } \\
\text { guidate dal docente } \\
\text { Analisi storica dei testi e dei } \\
\text { contesti } \\
\text { Ermeneutica interdisciplinare } \\
\text { Lavori di gruppo } \\
\text { Lezioni partecipative } \\
\text { Dibattito } \\
\text { Ricerca cooperativa in aula } \\
\text { Esercitazioni } \\
\text { Attività on-line } \\
\text { Apprendimento esperienziale } \\
\text { Uso di slide } \\
\text { Progetti co-costruiti con il } \\
\text { supporto del docente } \\
\text { Simulazioni }\end{array}$ & $\begin{array}{l}\text { Accertamento delle conoscenze } \\
\text { Verifica della comprensione concettuale } \\
\text { Valutazione di capacità applicative delle } \\
\text { conoscenze teoriche } \\
\text { Verifica della chiarezza espositiva e della } \\
\text { proprietà di linguaggio } \\
\text { Prova orale } \\
\text { Prova scritta } \\
\text { Esoneri } \\
\text { Modalità di iscrizione all'esame } \\
\text { Verifica di competenze nelle metodologie } \\
\text { di ricerca } \\
\text { Verifica dell'autonomia e della gestione } \\
\text { critica dello studente } \\
\text { Valutazione degli esiti di attività } \\
\text { laboratoriali } \\
\text { Questionario } \\
\text { Valutazione formativa } \\
\text { Prove di gruppo in itinere } \\
\text { Creazione di un intervento progettuale } \\
\text { Differenziazione delle domande } \\
\text { Verifica della capacità riflessiva } \\
\text { Esercitazioni }\end{array}$ \\
\hline LMG-01 Giurisprudenza & $\begin{array}{l}\text { Metodologia didattica } \\
\text { convenzionale } \\
\text { Lezioni frontali } \\
\text { Esercitazioni } \\
\text { Seminari disciplinari ed } \\
\text { interdisciplinari } \\
\text { Partecipazioni a Convegni } \\
\text { Incontri di studio tematici } \\
\text { Simulazione di casi } \\
\text { Uso di slide } \\
\text { Analisi e discussione di } \\
\text { sentenze } \\
\text { Analisi e discussione di casi } \\
\text { stranieri } \\
\text { Analisi e discussione di quesiti } \\
\text { giuridici }\end{array}$ & $\begin{array}{l}\text { Esoneri } \\
\text { Prova orale } \\
\text { Prova scritta } \\
\text { Prova scritta su traccia a scelta tra due } \\
\text { proposte } \\
\text { Accertamento delle conoscenze } \\
\text { Verifica della capacità analitica e } \\
\text { interpretativa di fonti antiche } \\
\text { Verifiche intermedie } \\
\text { Soluzione di un caso pratico } \\
\text { Verifica della padronanza linguistica } \\
\text { Valutazione di capacità applicative delle } \\
\text { conoscenze teoriche } \\
\text { Verifica della comprensione concettuale }\end{array}$ \\
\hline
\end{tabular}




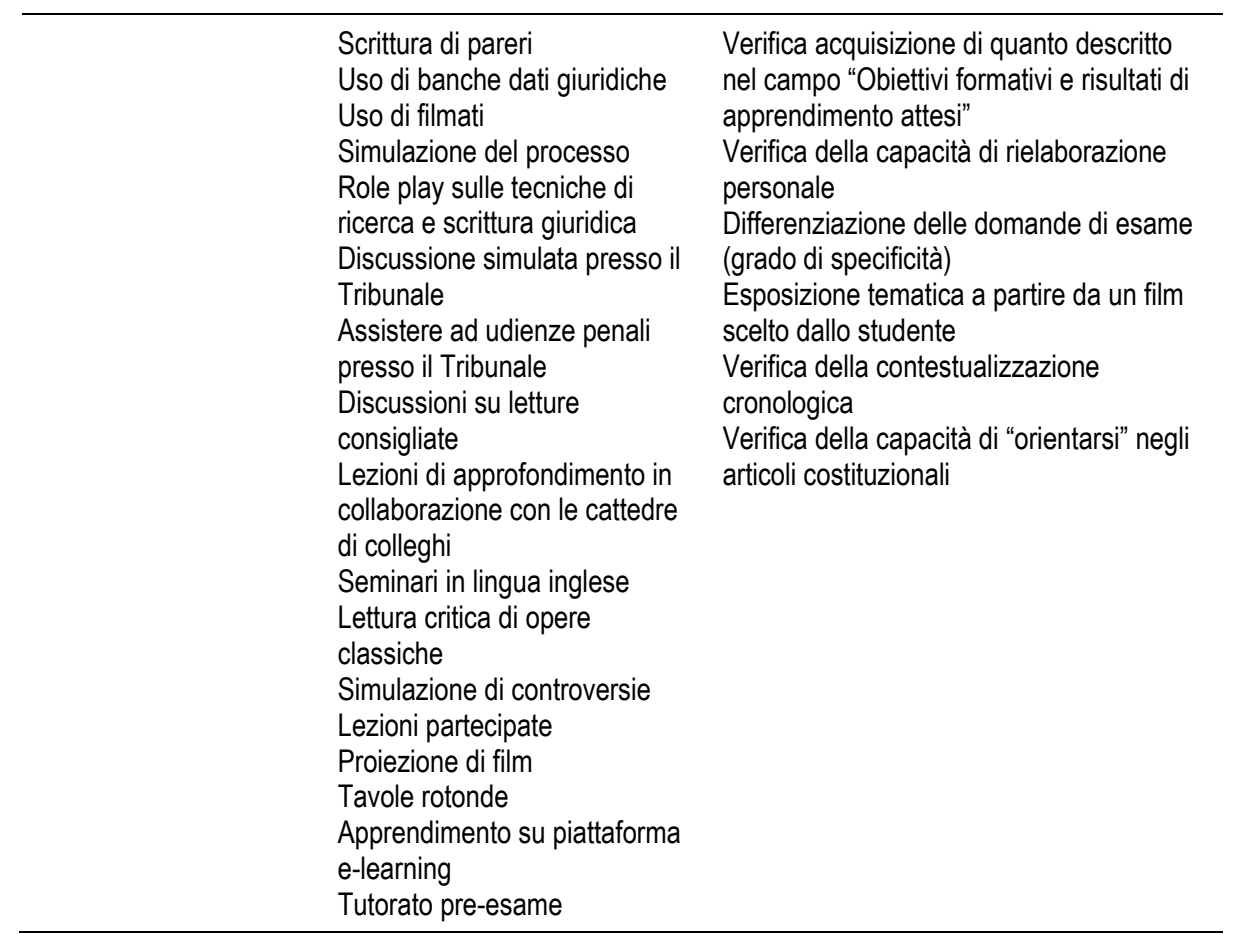

Oltre ad effettuare una codifica delle categorie emergenti relativamente ai campi documentali Metodi didattici e Valutazione, è stato anche "interrogato" l'intero corpus testuale attraverso la funzione Queries - modalità di esplorazione e di visualizzazione mediante grafici o diagrammi secondo precisi criteri di selezione - del software di analisi linguistica computazione NVivo.

Fra le funzioni Queries è stata utilizzata la Word Frequency, ossia una lista di parole-chiave ricorrenti, con indicazione della frequenza delle occorrenze (soglia minima: 5 occorrenze), preceduta da alcune azioni di preparazione del corpus testuale, quali l'omissione di alcuni lemmi (come le preposizioni o tutte le particelle brevi del discorso) e l'accorpamento dei sostantivi presenti al femminile/maschile o singolare/plurale. Il fine è stato quello di cercare di ricostruire una sorta di "trama semantico-concettuale" che sottende i Syllabi, quindi di comprendere quali parole ricorrono più frequentemente (Tabella 4). 
Tab. 4 - Word frequency (Count: 5 occorrenze): comparazione per Classe di laurea

\begin{tabular}{|c|c|c|}
\hline $\begin{array}{l}\text { Classe di } \\
\text { Laurea }\end{array}$ & $\begin{array}{l}\text { Parole co-occorrenti nella sezione } \\
\text { "Metodi" }\end{array}$ & $\begin{array}{l}\text { Parole co-occorrenti nella sezione } \\
\text { "Valutazione" }\end{array}$ \\
\hline L-30 Fisica & - & $\begin{array}{l}\text { saper (11), orale (6), campo (5), conoscere (5), } \\
\text { criteri (5), esplicitato (5), problemi (5), } \\
\text { valutazione (5) }\end{array}$ \\
\hline $\begin{array}{l}\text { L-19 Scienze } \\
\text { dell'Educazione e } \\
\text { della Formazione }\end{array}$ & $\begin{array}{l}\text { lezioni (17), frontali/e (17), seminari (11), } \\
\text { corso (10), approfondimento (9), attività } \\
\text { (5), gruppo (5) }\end{array}$ & $\begin{array}{l}\text { orale (15), studenti (13), valutazione (12), finale } \\
\text { (10), forma (9), esame (8), corso (7), scritta (7), } \\
\text { capacità (6), conoscenze (6), contenuti (6), } \\
\text { dovranno (6), frequentanti (6), prova (6), } \\
\text { colloquio (5), dimostrare (5), essere (5), } \\
\text { studentesse (5) }\end{array}$ \\
\hline $\begin{array}{l}\text { LM-85 Scienze } \\
\text { Pedagogiche }\end{array}$ & $\begin{array}{l}\text { frontali (11), lezioni (11), esercitazioni (6), } \\
\text { seminari/ seminariali (6) }\end{array}$ & $\begin{array}{l}\text { orale (14), valutazione (13), capacità (10), } \\
\text { conoscenza/e (9), corso (8), prova (7), itinere } \\
\text { (6), studente (6), previste/i (6), contenuti (5), } \\
\text { scritta (5) }\end{array}$ \\
\hline $\begin{array}{l}\text { LMG-01 } \\
\text { Giurisprudenza }\end{array}$ & $\begin{array}{l}\text { lezione/i (42), frontali (36), didattica (38), } \\
\text { convenzionale (33), metodologia ( } 31 \text { ), } \\
\text { seminari (16), casi (15), esercitazioni } \\
\text { (15), erogazione (14), modalità (14), } \\
\text { corso (12), discussione (11), esercitazioni } \\
\text { (11), seminari (11), studenti (9), } \\
\text { simulazione (8), studio (8), attività (7), } \\
\text { diritto (7), slides (7), multimediali (6), } \\
\text { pratici (6), processo (6), approfondimento } \\
\text { (5), metodo (5), PowerPoint (5), ricerca } \\
\text { (5), strumenti (5) }\end{array}$ & $\begin{array}{l}\text { esame (141), orale/i (86), valutazione (66), } \\
\text { voto (43), criteri (47), finale (40), studente (38), } \\
\text { capacità (34), conoscenze (31), programma } \\
\text { (24), intermedia (22), provale (22), parte/i (28), } \\
\text { verifica/che (20), Diritto (19), istituto/i (19), } \\
\text { abilità (18), frequentanti (18), studenti (18), } \\
\text { apprendimento (17), corso (17), domande (17), } \\
\text { accertare (15), formulazione (15), trentesimi } \\
\text { (15), modalità (14), espressa/o (14), } \\
\text { giuridicolila (14), acquisizione (12), esonero } \\
\text { (12), civile (11), preparazione (11), } \\
\text { processuale (11), acquisito (10), attesi (10), } \\
\text { conoscenza (10), diritto (10), formativi (10), } \\
\text { livello (10), obiettivi (10), risultati (10), verificare } \\
\text { (10), prevista (9), seconda (9), acquisite (8), } \\
\text { colloquio (8), contenuti (8), risposta/e (8), } \\
\text { critica/che (8), conto (8), scritto/e (8), } \\
\text { comprensione (7), descritte (7), espresso (7), } \\
\text { facoltativa (7), forma (7), previste (7), scritta } \\
\text { (7), sezione (7), sostenere (7), analisi (6), base } \\
\text { (6), casi (6), CFU (6), disciplina (6), intermedie } \\
\text { (6), idoneità (6), lezione (6), linguaggio (6), } \\
\text { Commerciale (6), esonero/i (6), metodi (6), } \\
\text { modalità (6), oggetto (6), penale (6), riferimento } \\
\text { (6), svolge (6), collocare (5), competenze (5), } \\
\text { descrizione (5), dettagliata (5), formulata (5), } \\
\text { accertamento (5), effettiva (5), particolare (5), } \\
\text { possibilità (5), prevedono (5), problematiche } \\
\text { (5), quesiti (5), relative (5), sistema (5), specifici } \\
\text { (5), terminologia (5), votazione (5) }\end{array}$ \\
\hline
\end{tabular}


Pur consapevoli che il numero di Syllabi analizzati determina largamente una differenziazione nel numero di parole co-occorrenti e che, per una comparazione adeguata sarebbe necessario considerare un numero equo, pari numericamente, di documenti, è possibile comunque fare qualche osservazione e ipotizzare eventuali indagini conoscitive di secondo livello, più esplicative.

La differenza sostanziale del corso LMG-01 Giurisprudenza rispetto agli altri (emergente dall'analisi soprattutto per numero di lemmi emergenti, in particolare riguardo al campo documentale relativo alla "Valutazione") si spiega solo in parte considerando il numero di documenti analizzati $(\mathrm{N}=49)$, per cui supponiamo un'ipotesi interpretativa che sarebbe interessante verificare successivamente, derivante dalla constatazione che oltre il $70 \%$ dei Syllabi di questo corso, a differenza degli altri, non è strutturato secondo i Descrittori di Dublino, ma in forma narrativa: è possibile che il format declinato secondo i Descrittori di Dublino possa avere un effetto "omologante" nella scrittura dei Syllabi e che una scrittura narrativa possa essere più efficace in termini di esplicitazione, più sintonica con le specificità linguistico-disciplinari o con il mondo della ricerca e percepita come più utile dai docenti? Che percezione hanno $i$ docenti della compilazione di un format documentale strutturato secondo Descrittori di Dublino? Quest'ipotesi trova una parziale conferma anche considerando alcuni esempi precedentemente analizzati (cfr. Tab. 2) di Syllabi strutturati secondo Descrittori di Dublino, in cui vengono richiamati solo i Descrittori, "vuoti", senza alcuna declinazione e contestualizzazione disciplinare: è possibile che l'indicazione dei Descrittori non sia sufficiente per un'efficace esplicitazione della progettazione didattica da parte del docente?

Indubbiamente queste prime considerazioni - al di là dell'ipotesi avanzata, tutta da verificare - mostrano con chiarezza la necessità di supportare i docenti, a livello formativo e tutoriale, nella compilazione dei Syllabi di insegnamento, in particolare nel saper coniugare i Descrittori di Dublino con la specificità del proprio insegnamento.

\section{Risultanze}

L'analisi dei Syllabi - condotta con l'intento di comprendere il grado di chiarezza e di eterogeneità nella strutturazione documentale di Ateneo, oltre che la coerenza fra obiettivi di insegnamento, risultati di apprendimento attesi, metodi di insegnamento, contenuti e modalità di verifica - ha reso evidenti alcune risultanze (in primis, alcune criticità nella compilazione documentale e 
aree di possibile miglioramento) capaci di orientare future prospettive di sviluppo sia a livello di indagine, che nell'ambito della formazione docente in università.

Come si è detto precedentemente, le "criticità" maggiori riguardano, in generale, i seguenti ambiti:

- l'alto grado di differenziazione dei modelli documentali;

- l'ambito della valutazione, con una evidente difficoltà di esplicitazione dei criteri;

- una centratura (in alcuni Syllabi) disciplinare, sul contenuto culturale, con assenza di attività learning-centered o student-centered (e sovrapposizione semantica fra Risultati di apprendimento previsti e Obiettivi di insegnamento);

- una scarsa cura documentale, ravvisabile in diversi Syllabi che presentano: indicazione non aggiornata dell' anno accademico, compilazione generica di alcuni campi documentali (soprattutto quella relativa ai Risultati di apprendimento previsti, spesso indicati in maniera incompleta, generica e vaga o confusi con gli Obiettivi di insegnamento), assenza di informazioni o di interi campi documentali (in primis i criteri di valutazione), duplicazione senza variazione dello stesso Syllabus per insegnamenti differenti di uno stesso insegnante;

- una difficoltà più evidente, nei Syllabi di matrice pedagogica, nell'esplicitare criteri e modalità di valutazione: un dato, questo, inatteso e meritevole di approfondimento, che lascia ipotizzare la difficoltà di "dire" con chiarezza ciò che forse viene sottinteso, dato per ovvio, in un certo ambito disciplinare.

Si riporta una tabella riepilogativa (Tabella 5) delle criticità emerse dalla comparazione di 94 Syllabi, condotta su 4 Corsi, 2 di area pedagogica e 2 di area disciplinare: 
Tab. 5 -Criticità emergenti dall'analisi documentale, differenziate per Classe di laurea

\begin{tabular}{|c|c|}
\hline SYLLABI DI AREA PEDAGOGICA & SYLLABI DI AREA DISCIPLINARE \\
\hline $\begin{array}{l}\text { L-19 Scienze dell'Educazione e della } \\
\text { Formazione }\end{array}$ & L30 Fisica \\
\hline $\begin{array}{l}\text { - } \text { eterogeneità della struttura documentale } \\
\text { - confusione/sovrapposizione fra campi } \\
\text { - } \text { documentali } \\
\text { - } \text { assenza dei criteri di valutazione } \\
\text { - compilazione generica dei Risultati di } \\
\text { apprendimento previsti, priva di } \\
\text { contestualizzazione disciplinare } \\
\text { - maggiore esplicitazione di criteri e modalità di } \\
\text { valutazione nei Syllabi disciplinaristi che in } \\
\text { quelli di area pedagogica }\end{array}$ & $\begin{array}{l}\text { - } \text { eterogeneità della struttura documentale } \\
\text { - } \quad \text { centratura sul contenuto disciplinare } \\
\text { - } \text { assenza di informazioni } \\
\text { - } \quad \text { assenza di campi documentali } \\
\text { - } \text { anno accademico non aggiornato } \\
\text { - difformità nei Risultati di apprendimento attesi } \\
\text { - (un terzo secondo Descrittori di Dublino) } \\
\text { - Risultati di apprendimento attesi poco definiti }\end{array}$ \\
\hline LM-85 Scienze Pedagogiche & LMG-01 Giurisprudenza \\
\hline $\begin{array}{l}\text { - assenza di campi documentali } \\
\text { - } \text { assenza di informazioni } \\
\text { - } \text { confusione/sovrapposizione fra campi } \\
\text { - compilazione generica dei Risultati di } \\
\text { apprendimento previsti, priva di } \\
\text { contestualizzazione disciplinare } \\
\text { - maggiore esplicitazione di criteri e modalità di } \\
\text { valutazione nei Syllabi disciplinaristi che in } \\
\text { quelli di area pedagogica }\end{array}$ & $\begin{array}{l}\text { - eterogeneità della struttura documentale } \\
\text { (prevalenza narrativa, no Dublin Descr.) } \\
\text { - Risultati di apprendimento previsti poco definiti } \\
\text { - centratura sul contenuto disciplinare } \\
\text { - confusione/sovrapposizione fra campi } \\
\text { documentali (in particolare fra Risultati di } \\
\text { apprendimento previsti e Obiettivi di } \\
\text { insegnamento) } \\
\text { - duplicazione (senza variazioni) dello stesso } \\
\text { Syllabus per insegnamenti differenti dello stesso } \\
\text { insegnante }\end{array}$ \\
\hline
\end{tabular}

L'analisi ha anche mostrato una eterogeneità nel numero di parole co-occorrenti, con una differenza sostanziale - per numero e tipologia di parole - per quanto riguarda il corso LMG-01 Giurisprudenza, di cui oltre il 70\% dei Syllabi non presenta una struttura secondo i Descrittori di Dublino, ma in forma narrativa. L'ipotesi interpretativa avanzata, che andrebbe verificata con eventuali ulteriori indagini, riguarda proprio il possibile impatto dei Descrittori di Dublino sul livello di esplicitazione e di chiarezza documentale: è possibile che una strutturazione più narrativa rispetto a quella individuata dai Descrittori di Dublino possa risultare più efficace e sintonica con le specificità linguistico-disciplinari e con le ricerche e gli studi condotti dagli stessi docenti?

Il dato "mancante", probabilmente uno dei limiti di questo primo lavoro di analisi, è la "voce" degli insegnanti: occorre certamente avviare ulteriori indagini conoscitive di secondo livello, che vadano ad indagare più in profondità le 
ragioni di certe scelte documentali e ad individuare, in maniera più mirata, $\mathrm{i}$ bisogni formativi dei docenti.

\section{Quali elementi utili alla redazione efficace del Syllabus di insegna- mento? Verso la formalizzazione di linee guida per il Syllabus co- disciplinare}

Il lavoro di analisi dei Syllabi ha mostrato la necessità di individuare metodi, strumenti e supporti affinché il docente possa esplicitare - con un adeguato livello di coerenza interna - le informazioni relative al proprio insegnamento: questo lavoro di esplicitazione è necessario non solo per chiarire agli studenti che cosa sia atteso da loro, ma anche per comunicare all'esterno (altri docenti, famiglie, stakeholders) la progettazione del proprio insegnamento (Moon, 2002; Serbati, 2019), in modo da specificare la relazione fra standard attesi dal singolo insegnamento e le competenze in uscita previste dal Corso di studi.

A tal fine, è importante individuare alcuni elementi utili alla redazione efficace di un Syllabus, alcuni suggeriti dagli esiti della stessa analisi condotta presso l'Università di Bari, altri desunti dalla letteratura e da materiale formativo elaborato ad hoc (cfr. slide elaborate da Anna Serbati, in collaborazione con Kate Riley, per la formazione dei docenti svoltasi il 16 ottobre 2018, pubblicate sul sito dell'Università di Bari):

- scegliere parole appropriate per dettagliare al meglio il dominio scientifico e la complessità dell'apprendimento atteso al termine dell'insegnamento, in modo da essere - più che conforme solo a format e standard - anche "fedele" alla specificità disciplinare del proprio insegnamento (evitando, come è emerso dall'analisi, l'utilizzo di espressioni vaghe, dell'utilizzo decontestualizzato dei Descrittori di Dublino) e, soprattutto, comprensibile in maniera chiara dagli studenti;

- progettare il Syllabus verificando la corrispondenza fra risultati di apprendimento, figura professionale in uscita, competenze relative e pertinenza del curriculum del CdS, anche per mezzo di strumenti specificatamente dedicati, come ad esempio la Matrice di incrocio tra obiettivi espressi tramite $i$ descrittori di Dublino e attività didattiche del corso di laurea, elaborata dall'Università di Padova (Serbati \& Zaggia, 2012);

- distinguere in modo chiaro i Risultati di apprendimento dagli Obiettivi di insegnamento, anche facendo riferimento a tassonomie (Anderson, Krathwohl et al., 2001), utili per classificare i domini dell'apprendimento in modo 
gerarchico, per creare un linguaggio comune e avere degli standard di livello su cui differenziare gli obiettivi di insegnamento;

- indicare i metodi, gli strumenti e i criteri di valutazione, dettagliando il più possibile tutte le informazioni utili per lo studente ai fini della valutazione finale (tipo di prova, strutturazione della prova, peso di eventuali verifiche intermedie, criteri valutativi ecc.) e in maniera il più possibile coerente con quanto indicato sia nei Risultati di apprendimento previsti che nei Metodi di insegnamento (Biggs, 2003);

- descrivere, oltre alle "responsabilità" del docente, quelle degli studenti, chiamati in primis ad auto-valutare la propria preparazione (O'Brien, Millis \& Cohen, 2008): in tal senso il Syllabus, come ben sottolinea Serbati (2019, p. 52), rappresenta un vero e proprio patto formativo con gli studenti che va ben al di là del mero adempimento burocratico.

A questi elementi vogliamo aggiungerne un ultimo, che riviene dalle interessanti differenze emerse dall'analisi comparativa fra Syllabi di diversa area disciplinare: l'importanza di dare vita, all'interno dei Teaching and Learning Center universitari, a Team interdisciplinari (Newell, 1994) composti da docenti di prospettive epistemologiche differenti che possono cooperare alla redazione del Syllabus, nella convinzione ormai matura che oggi abbia senso, anche in Università:

Parlare di dialettica da costruire sempre più sintonicamente fra la ricerca in didattica generale e la ricerca in ambito specificatamente disciplinare [...]: ovvero di uno spazio-tempo dell'azione (ove l'insegnante opera le sue continue regolazioni) come il "fondamento" su cui costruire l'interconnessione profonda fra l'epistemologia disciplinare (con i suoi statuti, le sue grammatiche) e la didattica generale (con il suo statuto e la sua grammatica) (Perla, Brusa \& Vinci, 2018, p. 128).

\section{Riferimenti bibliografici}

Anderson, L.W., Krathwohl, D.R., Airasian, P.W., Cruikshank, K.A., Mayer, R.E., Pintrich, P.R., Raths, J., \& Wittroch, M.C. (2001). A taxonomy for learning, teaching and assessing: A revision of Bloom's taxonomy of educational objectives. New York: Longman.

Beach, A.L., Sorcinelli, D.M., Austin, A.E., \& Rivard, J.K. (2016). Faculty Development in the Age of Evidence: Current Practices, Future Imperatives. Sterlin (VA): Stylus Pub Llc.

Biggs, J. (2003). Teaching for Quality Learning at University (2nd edition). Buckingham, UK: Society for Research into Higher Education \& OU Press. 
Bowen, G.A. (2009). Document Analysis as a Qualitative Research Method. Qualitative Research Journal, 9(2), 27-40.

Charmaz, K. (2006). Constructing grounded theory: A practical guide through qualitative analysis. Thousand Oaks, CA: Sage.

Damiano, E. (2013). La mediazione didattica. Per una teoria dell'insegnamento. Milano: FrancoAngeli.

Denzin, N. (2010). Moments, Mixed Method, and Paradigms Dialogs. Qualitative Inquiry, 16(6), 419-427.

DeZure, D., Van Note Chism, N., Sorcinelli, M.D., Cheong, G., Ellozy, A.R., Holley, M., Kazem, B. \& Atrushi, D. (2012). Building International Faculty-Development Collaborations: The Evolving Role of American Teaching Centers. The Magazine of Higher Learning, 44(3), 24-33.

European Commission (2013). High Level Group on the Modernisation of Higher Education. Report to the European Commission on Improving the quality of teaching and learning in Europe's higher education institutions. Luxembourg: Publications Office of the European Union.

European Network for Quality Assurance in Higher Education (ENQA) (2015). Standards and Guidelines for Quality Assurance in the European Higher Education Area (ESG). Brussels, Belgium: ENQA.

Felisatti, E., \& Serbati, A. (2015). Apprendere per imparare: formazione e sviluppo professionale dei docenti universali. Un progetto innovativo dell'Università di $\mathrm{Pa}$ dova. Giornale Italiano della Ricerca Educativa, 14, 323-339.

Felisatti, E., \& Serbati, A. (Eds.) (2017). Preparare alla professionalità docente e innovare la didattica universitaria. Milano: FrancoAngeli.

Gaebel, M., \& Zhang, T. (2018). Trends 2018: Learning and Teaching in the European Higher Education Area. European University Association.

Hussein, A. (2009). The use of triangulation in social sciences research: Can qualitative and quantitative methods be combined? Journal of Comparative Social Work, 1(1), 1-12.

Moon, J. (2002). The module \& programme development handbook: a practical guide to linking levels, learning outcomes \& assessment. London: Routledge.

Newell, W.H. (1994). Designing Interdisciplinary Courses. New Directions for Teaching and Learning, 58, 35-51.

O'Brein, J.G., Millis, B.J., \& Cohen, M.W. (2008). The Course Syllabus: A LearningCentered Approach, second edition. San Francisco: Jossey-Bass.

Perla, L. (2004). Valutazione e qualità in Università. Roma: Carocci.

Perla, L. (2011). L'eccellenza in cattedra. Dal saper insegnare alla conoscenza dell'insegnamento. Milano: FrancoAngeli.

Perla, L. (2018). Formare il docente alla didattica universitaria: il cantiere dell'innovazione. In M. Michelini (a cura di). Riflessioni sull'innovazione didattica universitaria. Interventi alla tavola rotonda GEO (79-88). Udine: Forum.

Perla, L., Brusa, A., \& Vinci, V. (2018). Insegnare il paesaggio storico. Tratteggio didattico co-epistemologico. Italian Journal of Educational Research, 9(20), 77-101. 
Perla, L., \& Vinci, V. (2018a). Dall'analisi dei bisogni formativi dei docenti universitari all'organizzazione del Teaching Learning Laboratory: la ricerca PRODID presso l'Università di Bari. Education Sciences \& Society, 2, 120-140.

Perla, L., \& Vinci, V. (2018b). TLL (Teaching Learning Laboratory) e formazione dialettica dei docenti universitari alla didattica: primi passi verso la certificazione della competenza pedagogica in Uniba. Lifelong Lifewide Learning, 15(32), 68-88.

Perla, L., \& Vinci, V. (2020). Follow up del progetto PRODID per lo sviluppo professionale dei docenti universitari: l'innovazione curricolare nel TLL e l'analisi dei Syllabi di insegnamento. In A. Lotti, \& P.A. Lampugnani (Eds.). Faculty Development e valorizzazione delle competenze didattiche dei Docenti nelle Università Italiane. Genova: GUP.

Saryon, A., \& Frenay, M. (Eds.) (2010). Building teaching capacities in higher education: A comprehensive international model. Sterling, VA: Stylus Publishing.

Serbati, A., \& Zaggia, C. (2012). Allineare le metodologie di insegnamento, apprendimento e valutazione ai learning outcomes: una proposta per i corsi di studio universitari. Giornale Italiano della Ricerca Educativa, 5(9), 11-26.

Serbati, A. (2019). Come definire i traguardi dell'apprendimento degli studenti: dagli obiettivi educativi alle competenze e Learning Outcomes. In A. Dipace \& V. Tamborra (Eds.). Insegnare in Università. Metodi e strumenti per una didattica efficace (37-54). Milano: FrancoAngeli.

Sorcinelli, M.D., Austin, A., Eddy, P. \& Beach, A. (2006). Creating the future offaculty development. San Francisco, CA: Jossey-Bass.

Strauss, A., \& Corbin, J. (1990). Basics of Qualitative Research: Grounded Theory Procedures and Techniques. Newbury Park: Sage.

Varma-Nelson, P., \& Turner, R. (2017). Faculty engagement with scholarly teaching and the culture and organization of a teaching and learning center. In E., Felisatti, A., Serbati (Eds.) Preparare alla professionalità docente e innovare la didattica universitaria (116-125). Milano: FrancoAngeli.

Villa Sanchez, A., \& Poblete Ruiz, M. (2008). Competence-based learning. Tuning Project, Bilbao: University of Deusto. 